\title{
Randomized controlled trials do not reflect reality: Real-world analyses are critical for treatment guidelines!
}

\author{
Martin T. R. Grapow, MD, ${ }^{a}$ Robert von Wattenwyl, MD, ${ }^{a}$ Ulrich Guller, MD, ${ }^{b}$ Friedhelm Beyersdorf, MD, PhD, ${ }^{c}$ \\ and Hans-Reinhard Zerkowski, MD
}

From the Divisions of Cardio-Thoracic Surgery ${ }^{\mathrm{a}}$ and General Surgery and Surgical Research, ${ }^{\mathrm{b}}$ University Hospital, Basel, Switzerland, and the Department of Cardiovasular Surgery, ${ }^{\mathrm{c}}$ University Hospital, Freiburg, Germany.

Received for publication Jan 6, 2006; revisions received March 6, 2006; accepted for publication March 15, 2006.

Address for reprints: Martin Grapow, MD, Division of Cardio-Thoracic Surgery, University of Basel, Spitalstrasse 21, CH-4031 Basel, Switzerland (E-mail: mgrapow@uhbs.ch).

J Thorac Cardiovasc Surg 2006;132:5-7

$0022-5223 / \$ 32.00$

Copyright $\odot 2006$ by The American Association for Thoracic Surgery

doi:10.1016/j.jtcvs.2006.03.035
A recent article by Hannan and coworkers ${ }^{1}$ including almost 60,000 patients from New York's cardiac registries forces us to reflect on the reliability of inferences made from randomized controlled trials (RCTs). The same applies to the treatment decisions exclusively derived from findings of RCTs for patients with coronary heart disease under everyday conditions.

Since the mid-1980s, surgical revascularization has become a standardized procedure. Since then, the coronary artery bypass grafting $(\mathrm{CABG})$ technique has continuously improved. ${ }^{2}$ The superiority of surgical revascularization over medical therapy was documented at an early stage. ${ }^{3}$

Since the introduction of percutaneous transluminal coronary angioplasty and stents, percutaneous coronary intervention (PCI) has become a therapeutic alternative to medical and surgical therapy in many patients with coronary artery disease. There has been growing demand for, and use of, PCI because it is less invasive, so that today it is carried out 3 times more frequently than coronary surgery. ${ }^{4}$

Over the last 10 years, numerous prospective RCTs have been conducted to answer the question of whether and in what situations CABG or PCI results in a better medium- and long-term course. Until very recently, the focus of research has been exclusively on RCTs and meta-analyses, whereas large population-based studies or cardiac registry analyses have received less attention.

The value of stringently conducted RCTs is undisputed because they have great internal validity. However, the crucial question is whether their results have relevance to everyday decision making. In RCTs patients are randomly assigned to standard and investigational arms and are followed up over a defined period. The final results of the randomized groups are often compared, irrespective of whether the positive result of one treatment arm was induced in part by using the alternative treatment principle (the intent-to-treat principle) as a result of crossover. Although the intent-to-treat analysis represents a central tenet of RCTs, it might bias the results versus no difference in the context of a comparison of PCI versus CABG. Moreover, it is not an uncommon phenomenon that RCTs are underpowered. ${ }^{5,6}$

Clearly, even the most thoroughly planned and well-executed investigation might fail to answer the research question if the sample size is too small. If an underpowered study does not find statistically significant differences, it is unclear whether there is truly no difference between the treatment options or whether the sample size was prohibitively small to provide sufficient evidence for a statistically significant difference. ${ }^{6,7}$

Finally, patients are usually recruited on the basis of stringent inclusion and exclusion criteria that are often worryingly dissimilar to the overall patient population with coronary artery disease. Meta-analyses ${ }^{8}$ do not resolve this problem because they are based on those studies just described.

This high degree of patient selection represents the crucial limiting factor in many randomized clinical trials; the patients included in such studies seldom reflect the population encountered in routine practice. According to the analysis carried out by Brett and colleagues, ${ }^{7}$ many RCTs included markedly less than $10 \%$ of all screened patients. Indeed, patients with common-sense indications for CABG and contraindications for PCI (eg, left main stenosis or diffuse, severe 3-vessel diseases) 
are often precluded from entering an RCT because the premise of an RCT is that all patients benefit equally from both procedures.

By including a population that is known not to benefit from $\mathrm{CABG}^{9}$ and by excluding the complex cases that predominantly constitute the population undergoing $\mathrm{CABG}$ nowadays, these trials were definitely biased against $\mathrm{CABG}$ operations and toward the performance of PCI. ${ }^{10}$

However, it is striking throughout the literature that the mean number of grafts-peripheral anastomoses per patient is usually higher than the mean number of stents per patient. It can thus be concluded that the criterion "completeness of revascularization," which is unquestionably important for the prognosis, is in fact not achieved as a rule in these highly selected RCT patient populations.

If the majority of patients are excluded from participating in RCTs on the basis that they cannot undergo equivalent interventional treatment, it follows that the study results and conclusions are exclusively valid for patients with identical inclusion criteria as those in RCTs. In particular, the finding that mortality did not increase in patients undergoing PCI compared with in those who had undergone CABG in these RCTs only applies in a clinical setting to patients with profiles included in the study. However, this finding has often been extended to patients who would actually have been excluded from the study according to the RCT protocol.

Cohort studies and registry analysis studies try to bridge this gap in knowledge. Population-based studies attempt to derive more general conclusions from the results obtained in a nonselected patient population. Brener and associates ${ }^{11}$ compared the clinical course of patients undergoing CABG with that of patients undergoing PCI at the Cleveland Medical Center. These patients had been treated with PCI or an operation, depending on the advice of the managing physician. With regard to their comorbidity, the groups treated with an operation and those treated with PCI differed significantly, with the former being more comorbid. PCI was associated with significantly higher mortality rates (riskadjusted hazard ratio, 2.3; 95\% confidence interval, 1.92.9). The results of this population investigated intensively at a single institution have been confirmed in a real-world study by Hannan and coworkers ${ }^{1}$ by analyzing 59,314 patients in New York's cardiac registries who underwent either CABG or PCI (all with stents) from 1997 through 2000. Risk adjustment was performed by using both multivariable and propensity-score analysis.

The fundamental difference between multivariable and propensity-score analysis is that the former adjusts for confounders focusing on the relationship between baseline characteristics (eg, age, sex, and comorbidities) and the outcome (overall survival), whereas propensity-score analysis focuses on the relationship between baseline character- istics and the primary predictor variable (CABG vs $\mathrm{PCI}$ ). Hence propensity-score analysis attempts to reconstruct a situation (case matching) similar to randomization. ${ }^{12}$

The major limitation of observational studies is that both propensity-score and multivariable analysis can exclusively adjust for known confounders. Neither propensity-score nor multivariable analysis can risk adjust for unobserved or unknown confounders. ${ }^{12}$ This represents an important advantage of RCTs that distribute both known and unknown confounders equally to both study arms. ${ }^{13}$ Further limitations of observational studies based on registries might include potential coding errors and missing data. ${ }^{13}$

In the investigation by Hannan and coworkers, ${ }^{1}$ patients with 2- and 3-vessel disease undergoing CABG had a significantly better long-term overall survival, as well as a lower risk of reintervention, after risk adjustment using both multivariable and propensity-score analysis. Interestingly, in patients with 3-vessel disease, CABG was associated with a significantly lower mortality rate compared with that of PCI, even without risk adjustment. Equivalence with respect to prognosis between PCI and CABG was found only in patients with 2-vessel disease with or without proximal left anterior descending coronary artery involvement in unadjusted survival analyses.

Overall, PCI was performed in $61 \%$ of patients with double-vessel disease, whereas only $42 \%$ with proximal left anterior descending coronary artery disease and 14\% with triple-vessel disease were treated with PCI. On the basis of these results, one can conclude that physicians generally make good choices for their patients.

The investigation by Hannan and coworkers ${ }^{1}$ is particularly valuable because it reflects the real-world situation, being based on a large and unselected group of patients in whom the indications for CABG and PCI, respectively, were identified as part of a day-to-day routine. This analysis does not contradict the large numbers of RCTs comparing PCI versus CABG but applies to a much broader patient population. If patients do not meet the inclusion criteria of RCTs, it seems advisable to decide on therapy by referring to large registry analyses or cohort studies. Considering the RCTs available today, ${ }^{7}$ there is essentially no significant difference in survival for one of the 2 strategies in the medium- and long-term course. However, surgical intervention is associated with a significantly lower reintervention rate, a greatly reduced recurrence of angina, and a higher rate of complete revascularization.

It is interesting that this synoptic statement is also reflected in clinically unselected studies. Van Domburg and colleagues, ${ }^{14}$ at the Rotterdam Thorax Center, compared patient profiles and treatment data, as well as PCI and CABG outcomes, in the same institution before and after introduction of drug-eluting stents. Despite a much higher proportion of 3-vessel disease (75\% compared with $22 \%$ ), 
diabetes (24\% compared with $17 \%$ ), and much worse left ventricular ejection fraction in the $\mathrm{CABG}$ group in the drug-eluting stent era, the mortality of $2.2 \%$ within 12 months after CABG compared favorably with the mortality of $4.1 \%$ in the PCI group.

In summary, only by considering both results from RCTs and large cohort studies can real-life clinical situations be reflected to a degree capable of providing applicable treatment guidelines useful in a daily clinical setting.

\section{References}

1. Hannan EL, Racz MJ, Walford G, Jones RH, Ryan TJ, Bennett E, et al. Long-term outcomes of coronary-artery bypass grafting versus stent implantation. $N$ Engl J Med. 2005;352:2174-83.

2. Loop FD, Lytle BW, Cosgrove DM, Stewart RW, Goormastic M, Williams GW, et al. Influence of the internal-mammary-artery graft on 10-year survival and other cardiac events. $N$ Engl J Med. 1986;314: $1-6$.

3. Eleven-year survival in the Veterans Administration randomized trial of coronary bypass surgery for stable angina. The Veterans Administration Coronary Artery Bypass Surgery Cooperative Study Group. N Engl J Med. 1984;311:1333-9.

4. Bruckenberger E. Herzbericht 2003 mit Transplantationschirurgie. In: Bruckenberger E, editor. 16th ed. Hannover: 2004.

5. Freiman JA, Chalmers TC, Smith H Jr, Kuebler RR. The importance of beta, the type II error and sample size in the design and interpre- tation of the randomized control trial. Survey of 71 "negative" trials. N Engl J Med. 1978;299:690-4.

6. Guller U, Oertli D. Sample size matters: a guide for surgeons. World J Surg. 2005;29:601-5.

7. Brett W, Hirschmann MT, Guller U, Zerkowski H-R. CABG vs. PCI in coronary artery disease: what is the evidence? Card Surg Today. 2005;2:43-55.

8. Hoffman SN, TenBrook JA, Wolf MP, Pauker SG, Salem DN, Wong JB. A meta-analysis of randomized controlled trials comparing coronary artery bypass graft with percutaneous transluminal coronary angioplasty: one- to eight-year outcomes. J Am Coll Cardiol. 2003; 41:1293-304.

9. Yusuf S, Zucker D, Peduzzi P, Fisher LD, Takaro T, Kennedy JW, et al. Effect of coronary artery bypass graft surgery on survival: overview of 10-year results from randomised trials by the Coronary Artery Bypass Graft Surgery Trialists Collaboration. Lancet. 1994; 344:563-70

10. Taggart DP. Surgery is the best intervention for severe coronary artery disease. BMJ. 2005;330:785-6.

11. Brener SJ, Lytle BW, Casserly IP, Schneider JP, Topol EJ, Lauer MS. Propensity analysis of long-term survival after surgical or percutaneous revascularization in patients with multivessel coronary artery disease and high-risk features. Circulation. 2004;109:2290-5.

12. Adamina M, Guller U, Weber WP, Oertli D. Propensity score and the surgeon. Br J Surg. 2006. Epub ahead of print.

13. Guller U, Surgical outcomes research based on administrative data: inferior or complementary to prospective randomized clinical trials? World J Surg. 2006;30:255-66.

14. van Domburg RT, Lemos PA, Takkenberg JJ, Liu TK, van Herwerden LA, Arampatzis CA, et al. The impact of the introduction of drugeluting stents on the clinical practice of surgical and percutaneous treatment of coronary artery disease. Eur Heart J. 2005;26:675-81.

\section{Online-www.aats.org}

Now you can get The Journal of Thoracic and Cardiovascular Surgery online. The Journal online brings you faster delivery time, easy searching of current and back issues, links to PubMed, AATS, WTSA, and other important sites, and more. Visit the Journal online today.

\section{Receive tables of contents by e-mail}

To receive the tables of contents by e-mail, sign up through our Web site at http://journals.elsevierhealth.com/periodicals/ymtc

Choose E-mail Notification

Simply type your e-mail address in the box and click the Subscribe button. You will receive an e-mail to confirm that you have been added to the mailing list.

Note that TOC e-mails will be sent out when a new issue is posted to the Web site. 